\title{
Modification of the exchange bias effect by He ion irradiation
}

\author{
A. Mougin, T. Mewes, R. Lopusnik, M. Jung, D. Engel, A. Ehresmann, H. Schmoranzer, \\ J. Fassbender ${ }^{\mathrm{a}}$, and B. Hillebrands \\ Fachbereich Physik and Forschungs- und Entwicklungsschwerpunkt Materialwissenschaften, Universität Kaiserslautern, Erwin-Schrödinger- \\ Strasse, 67663 Kaiserslautern, Germany
}

$\mathrm{FeNi} / \mathrm{FeMn}$ exchange bias samples with a large exchange bias field at room temperature have been prepared on a $\mathrm{Cu}$ buffer layer. Upon irradiation with $\mathrm{He}$ ions, both the exchange bias field and the coercive field are modified. For low ion doses the exchange bias field is enhanced by nearly a factor of 2. Above a threshold dose of $0.3 \cdot 10^{15}$ ions $/ \mathrm{cm}^{2}$, the exchange bias field decreases continuously as the ion dose increases. The observed modifications are explained in terms of defect creation acting as pinning sites for domain walls and atomic intermixing.

\section{I) Introduction}

In the last few years, there has been a large interest in studying ferromagnetic / antiferromagnetic bilayers. To date, the microscopic origin of the exchange bias effect is still under discussion. The use of ion irradiation appears to be a good tool to influence and hence investigate the role of atomic defects on the exchange bias field.

Recently, ion irradiation has been shown to induce structural modifications in ultrathin film or multilayer systems. In $\mathrm{Co} / \mathrm{Pt}$ multilayers, it was possible to change the easy direction of magnetization from perpendicular to in-plane with respect to the film plane, due to interface intermixing [1], [2]. In contrast, for FePt alloy films, a chemical ordering of the alloy occurs after ion irradiation [3]. From the application's point of view, the interest in ion irradiation of magnetic films results from the possibility to pattern these films magnetically on a length scale below 50 $\mathrm{nm}$, without modifying the sample topography [2].

The magnetic hysteresis loop of a thin ferromagnetic film (F) in direct contact to an antiferromagnetic film (AF) is modified compared to the one of a single ferromagnetic film. If such a bilayer is prepared in an applied magnetic field, or when it is heated above the Néel temperature of the AF film (which has to be lower than the Curie temperature of the F film) and subsequently cooled in a magnetic field, the spin arrangement in the AF layer contains information about the magnetization direction in the $\mathrm{F}$ layer. The exchange interaction at the interface acts as an internal field and the magnetization reversal of the $\mathrm{F}$ layer is shifted by the so-called exchange bias field, $H_{\mathrm{eb}}$. This effect has been known for a long time [4] and a number of different models [5]-[9] have been proposed to understand its nature. All models have in common that the exchange bias effect is of interfacial origin and therefore very sensitive to the microstructure of the bilayer and its interface. Hence small structural modifications may cause considerable changes in $H_{\mathrm{eb}}$ and the coercive field, $H_{\mathrm{c}}$.

Recently, it has been reported that both the exchange bias field and the coercive field can be reduced by ion irradiation in a controlled manner by adjusting the dose and the energy of the ions [10]. In addition, and first reported in this paper, not only a reduction but also an enhancement of $H_{\mathrm{eb}}$ can be induced: an increase of $H_{\mathrm{eb}}$ of nearly a factor of 2 is achieved in the low dose regime around $10^{14}$ ions $/ \mathrm{cm}^{2}$, for $\mathrm{FeNi} / \mathrm{FeMn}$ systems grown onto a $\mathrm{Cu}$ buffer layer. Above a threshold dose of $0.3 \cdot 10^{15}$ ions $/ \mathrm{cm}^{2}, H_{\mathrm{eb}}$ decreases continuously with the successive increase of the ion dose.

\section{II) Exchange-bias in FeNi/FeMn bilayers}

In order to investigate the influence of ion irradiation, we chose the well known FeNi/FeMn exchange bias system. The samples were prepared in a UHV-system with a base pressure of 5.10-10 mbar. Onto a thermally oxidized $\mathrm{Si}$ substrate with a $35 \mathrm{~nm} \mathrm{Cu}$ buffer layer, a $5 \mathrm{~nm}$ $\mathrm{Fe} 0.19 \mathrm{Ni0} .81$ layer (F) and a $10 \mathrm{~nm} \mathrm{Fe} 0.5 \mathrm{Mn} 0.5$ (AF) layer were grown. Finally, a $2 \mathrm{~nm} \mathrm{Cr}$ layer was deposited to prevent the samples from oxidation. All layers were grown at room temperature. After the preparation, the samples were heated and subsequently cooled in an ap-

\footnotetext{
a) author to whom correspondence should be addressed: e-mail: fassbend@physik.uni-kl.de

Preprint server AG-Hillebrands. http://haktar.physik.uni-kl.de/w_hilleb, submitted to IEEE Trans. Magn.
} 
plied magnetic field of 500 Oe to below the Néel temperature.

The magnetic properties were investigated ex situ by laterally resolved longitudinal magneto-optical Kerr-effect (MOKE) measurements at room temperature. The exchange bias field and the coercive field were found to be homogeneous across each sample. First, the samples were characterized prior any irradiation process. Figure 1a) shows a typical hysteresis loop of the exchange bias system. The initial exchange bias field, $H_{\text {eb,initial }}$, is as high as 190 Oe. This corresponds to an interface exchange energy of $0.08 \mathrm{erg} / \mathrm{cm} 2$. The respective coercive field $H_{\mathrm{c}}$ is near 22 Oe, which is considerably higher compared to simple FeNi films. An analysis of the hysteresis curves as a func-

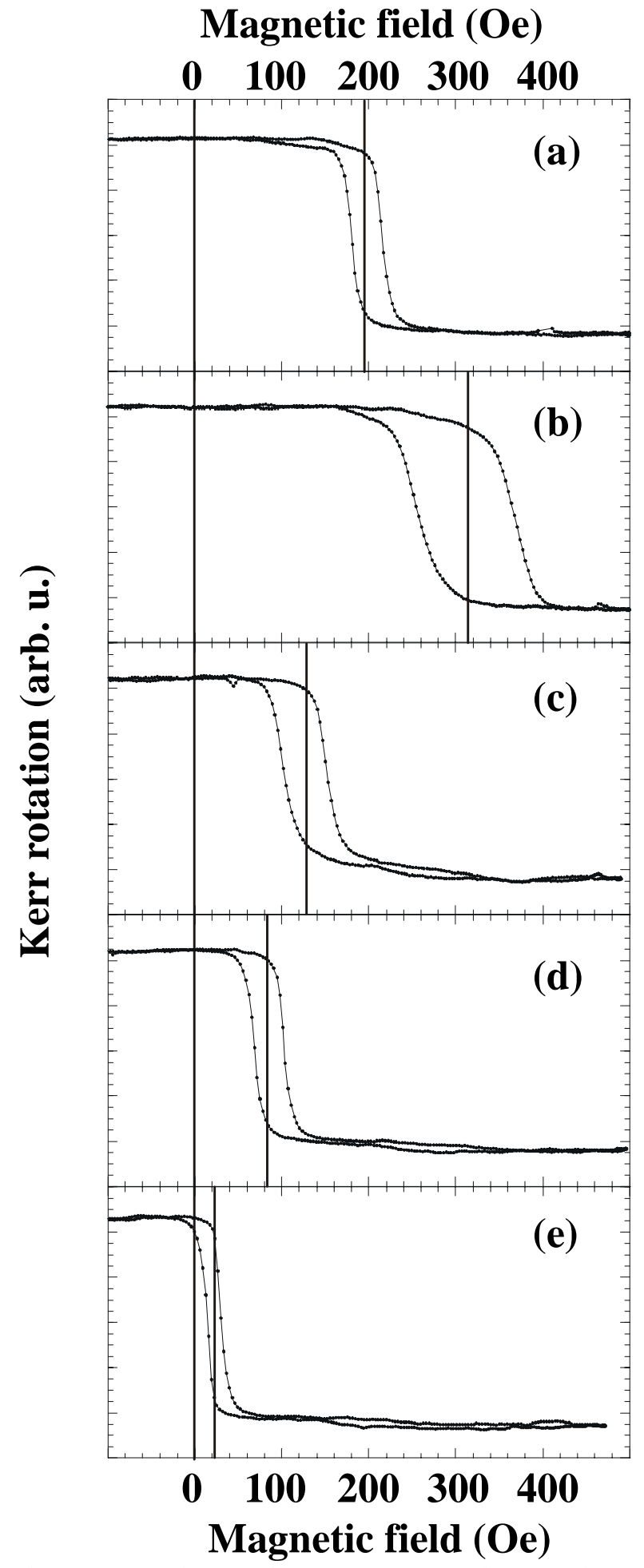

Figure 1: Hysteresis curves for $\mathrm{Cr} / \mathrm{Fe}_{0.5} \mathrm{Mn}_{0.5} / \mathrm{Fe}_{0.19} \mathrm{Ni}_{0.81} / \mathrm{Cu}$ exchange bias samples measured at room temperature. In a) the hysteresis curve of a sample prior to ion irradiation is shown. In b) - e) hysteresis curves for different ion doses are presented: b) $0.16 \cdot 10^{15}$ ions $/ \mathrm{cm}^{2}$, c) $1.43 \cdot 10^{15}$ ions $/ \mathrm{cm}^{2}$, d) $1.90 \cdot 10^{15}$ ions $/ \mathrm{cm}^{2}$, and e) $4.75 \cdot 10^{15}$ ions $/ \mathrm{cm}^{2}$. tion of the in-plane angle (not shown here) shows a unidirectional anisotropy, as expected for this system due to the symmetry implied by the exchange bias effect.

The large value of $H_{\mathrm{eb}}$ is likely caused by the $\mathrm{Cu}$ buffer layer. Therefore, an enlarged amount of (111) textured $\gamma$ FeMn crystallites is required to optimize $H_{\mathrm{eb}}$ in FeNi/FeMn systems [11], [12]. A Cu buffer layer has been shown either to promote [13] or to impede [14] the required texture. Detailed investigations on the structural properties are currently performed in order to clarify these different observations.

\section{III) Influence of ion irradiation}

After the initial magnetic characterization, the samples were inserted into an ion optical bench to perform the ion irradiation. He ions were produced in a Penning type source and accelerated by $10 \mathrm{kV}$. Different doses on the samples were realized by adjusting the beam current $(5-60 \mathrm{nA})$, depending on the accelerating voltage, and varying the irradiation time ( $3 \mathrm{~s}-120 \mathrm{~s})$ correspondingly, covering the ion dose range from $10^{13}$ ions $/ \mathrm{cm}^{2}$ to $6 \cdot 10^{15}$ ions $/ \mathrm{cm}^{2}$. Different ion doses were applied on one single sample by irradiating different areas of the sample. Hysteresis loops were measured along a lateral scan across the sample through the centers of the irradiated areas.

Figure 1 shows typical hysteresis loops collected at room temperature from samples irradiated with different ion doses. The curve presented in a) (non irradiated area) has been discussed in detail above. As a result of the ion irradiation process, $H_{\mathrm{eb}}$ and $H_{\mathrm{c}}$ are modified $(\mathrm{b}-\mathrm{e})$. In order to analyze this behavior more quantitatively, the normalized exchange bias field ratio, $H_{\mathrm{eb}} / H_{\text {eb,initial }}$, (a) and coercive field ratio $H_{\mathrm{c}} / H_{\mathrm{c} \text {,initial }}$ (b) are plotted in Fig. 2 as a function of ion dose. Two different regimes are identified: i) A pronounced increase of $H_{\mathrm{eb}}$ and $H_{\mathrm{c}}$ is found in the dose regime between $10^{13}$ and $0.3 \cdot 10^{15}$ ions $/ \mathrm{cm}^{2}$. This region of interest is presented enlarged in the inset of Fig. 2. The maximum values are found to be $H_{\mathrm{eb} \text {, max }}=315 \mathrm{Oe}$ and $H_{\mathrm{c}, \max }=56$ Oe. ii) For doses above $0.3 \cdot 10^{15}$ ions $/ \mathrm{cm}^{2}$ the evolution is reversed and $H_{\mathrm{eb}}$ is reduced. The shift of the hysteresis loops decreases continuously with succes-

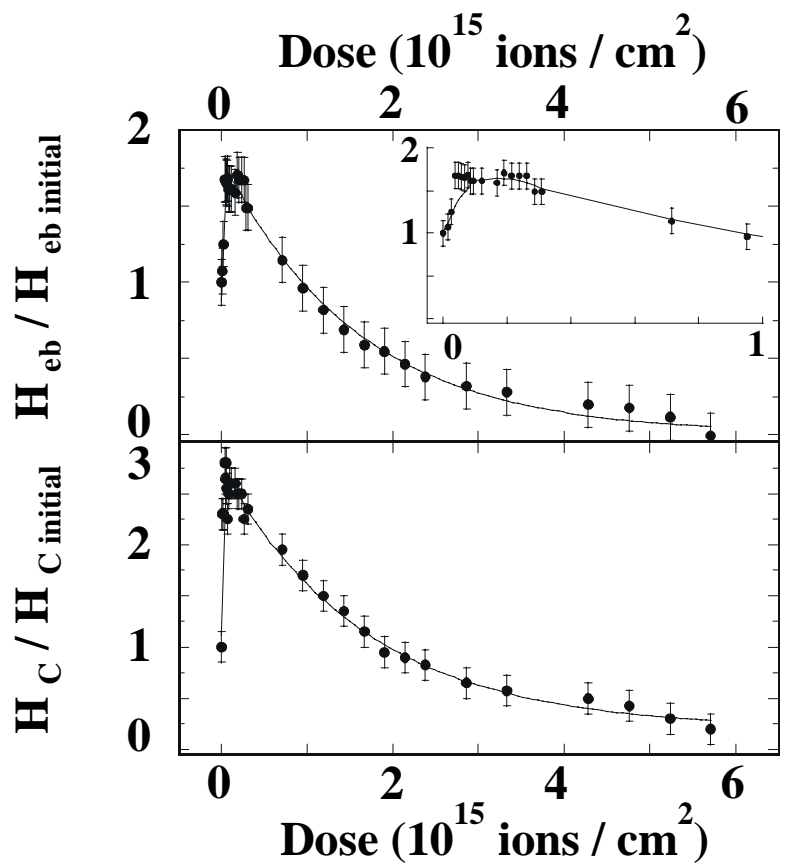

Figure 2: The exchange bias field ratio $H_{\mathrm{eb}} / H_{\mathrm{eb}, \text { initial }}$ (a) and the coercive field ratio $H_{\mathrm{c}} / H_{\mathrm{c} \text {,initial }}(\mathrm{b})$, extracted from the corresponding hysteresis curves, are plotted as a function of ion dose. The dose range below $10^{15}$ ions $/ \mathrm{cm}^{2}$ is presented on an enlarged scale in the inset. 
sive increase of the ion dose. Finally, the exchange bias is fully suppressed and the coercive field is reduced correspondingly. Note that the magnitude of the Kerr rotation is not affected by the ion irradiation until full suppression of the exchange bias. This indicates that the irradiation process does not modify the magnetic properties of the $F$ layer, up to high doses.

In order to understand the experimental results, the penetration depth and the energy loss mechanisms of the $\mathrm{He}$ ions have to be considered. The stopping of ions is generally separated into the electronic and the nuclear stopping power [15]. For heavy ions, depending on the target, the maximum of the nuclear stopping contribution occurs typically at energies of about $100 \mathrm{keV}$ whereas the electronic stopping contribution is dominant at $\mathrm{MeV}$ energies. However, the energy loss of very light, low energy ions in heavy materials exhibits a different behavior. In this case, both energy loss mechanisms have to be taken into account: ballistic collisions which lead to irreversible damage of the structure of the sample and inelastic scattering which results in electronic excitations [15], [16]. The average penetration depth of $\mathrm{He}$ ions accelerated with 10 $\mathrm{kV}$ is about $90 \mathrm{~nm}$ [15], which is much larger than the total thickness of the exchange bias system. Hence, the nuclear damage cascade is located in the substrate.

For low doses, the density of displaced atoms and the number of created defects are low in the F and AF layers. Although the as-prepared samples already exhibit a large exchange bias field, an enhancement by nearly a factor of 2 is observed for low doses. In order to understand this enlargement, we have to revisit the different models of the exchange bias effect [5]-[9]. It is generally accepted that exchange bias is due to the interactions between the $F$ and AF moments across the interface. Most models [5], [6] claim that $H_{\mathrm{eb}}$ results from an uncompensated magnetization at the interface [17] and the existence of domain walls parallel to the interface within the AF layer involving several layers of AF moments, which have recently been observed experimentally in $\mathrm{FeNi} / \mathrm{FeMn}$ systems [18]. In order to understand a large value of the exchange bias field, numerous pinning sites and thus AF domain walls are required [6]. Small doses of He ions create a low density of displaced atoms. Thereby $H_{\mathrm{eb}}$ is increased in the low dose regime. The observed increase of $H_{\mathrm{c}}$ is also consistent with the model. Our results are in full agreement with recent studies by Miltenyi et al., who showed that an increase of $H_{\mathrm{eb}}$ is caused by the creation of pinning sites in the AF layer generated by non magnetic defects [19].

A further increase of the ion dose leads to an enhanced density of defects. Subsequently, an increase of the interface roughness and eventually intermixing occur. Hence the exchange coupling between the $\mathrm{F}$ and $\mathrm{AF}$ layers is reduced, which leads to a decrease of $H_{\mathrm{eb}}$ [10]. In this regime, the reduction of the exchange coupling due to intermixing surpasses the effect of the pinning sites in the AF layer.

\section{IV) Conclusion}

$\mathrm{Fe}_{0.19} \mathrm{Ni}_{0.81} / \mathrm{Fe}_{0.5} \mathrm{Mn}_{0.5}$ bilayers have been grown on a $\mathrm{Cu}$ buffer layer. After the initial magnetic preparation, the asgrown samples exhibit a large exchange bias field of $H_{\mathrm{eb}}=190 \mathrm{Oe}$ and an enhanced coercive field of $H_{\mathrm{c}}=22$
Oe at room temperature. Both values can be tailored by He ion irradiation. For low ion doses an enhancement to the maximum values of $H_{\mathrm{eb}}=315 \mathrm{Oe}$ and $H_{\mathrm{c}}=56 \mathrm{Oe}$ is found at room temperature. For higher doses a reduction of both values is found consistent with prior investigations [10].

From the technological point of view, it is important to note that the magnetic properties can be tailored after device fabrication. Moreover, the use of ion irradiation for magnetic patterning purposes without changing the sample topography is of great importance. Since the ion beam is collimated close to the surface and the major part of the damage cascade is located in the substrate, proximity effects are negligible and patterning becomes possible down to the smallest lateral sizes feasible by focused ion beam directly or, alternatively, by the resolution of mask techniques [20].

\section{Acknowledgments:}

We thank G. Güntherodt and his group for communicating their results prior to publication. This work was partly supported by the EC-TMR program "Dynaspin" No. FMRX-CT97-0124.

\section{References}

[1] C. Chappert, H. Bernas, J. Ferre, V. Kottler, J.-P. Jamet, Y. Chen, E. Cambril, T. Devolder, F. Rousseaux, V. Mathet, and H. Launois, Science 280, 1919 (1998).

[2] T. Devolder, C. Chappert, Y. Chen, E. Cambril, H. Bernas, J. P. Jamet, and J. Ferre, Appl. Phys. Lett. 74, 3383 (1999).

[3] D. Ravelosona, C. Chappert, V. Mathet, and H. Bernas, Appl. Phys. Lett. 76, 236 (2000).

[4] W. H. Meiklejohn and C. P. Bean, Phys. Rev. 102, 1413 (1956); 105, 904 (1957).

[5] D. Mauri, H. Siegmann, P. S. Bagus, and E. Kay, J. Appl. Phys. 62, 3047 (1987).

[6] A. P. Malozemoff, J. Appl. Phys. 63, 3874 (1988).

[7] N. C. Koon, Phys. Rev. Lett. 78, 4865 (1997).

[8] T. C. Schulthess, and W. H. Butler, Phys. Rev. Lett. 81, 4516 (1998).

[9] L. Néel, Ann. Phys. (Paris) 2, 61 (1967).

[10] T. Mewes, R. Lopusnik, J. Fassbender, B. Hillebrands, M. Jung, D. Engel, A. Ehresmann, and H. Schmoranzer, Appl. Phys. Lett. in press, tentatively scheduled Feb. 21, 2000.

[11] R. Jungblut, R. Coehoorn, M. T. Johnson, Ch. Sauer, P. J. van der Zaag, A. R. Ball, Th. G. Rijks, J. aan de Stegge, and A. Reinders, J. Magn. Magn. Mat. 148, 300 (1995).

[12] S. Nakagawa, K. Nishimura, Y. Shimizu, and M. Naoe, J. Appl. Phys. 85, 4934 (1999)

[13] R. Nakatani, K. Hoshino, S. Noguchi, and Y. Sugita, Jap. J. Appl. Phys. 33, 133 (1994).

[14]C. Tsang, N. Heiman, K. Lee, J. Appl. Phys. 52, 2471 (1981).

[15] SRIM 2000 code, J. F. Ziegler, J. P. Biersack, and U. Littmark, The Stopping and Range of Ions in Solids, Pergamon, New York, Oxford (1985).

[16] P. Haussalo, K. Nordlund, and J. Keinonen, Nucl. Instr. Phys. Rev. B 111, 1(1995).

[17] W. J. Antel, Jr., F. Perjeru, and G. R. Harp, Phys. Rev. Lett. 83, 1439 (1999).

[18] V. I. Nikitenko, V. S. Gornakov, A. J. Shapiro, R. D. Schull, K. Liu, S. M. Zhou, and C. L. Chien, Phys. Rev. Lett. 84, 765 (2000).

[19] P. Miltenyi, M. Gierlings, J. Keller, B. Beschoten, G. Güntherodt, U. Nowak, K. D. Usadel, to be published.

[20] B. D. Terris, L. Folks, D. Weller, J. E. E. Baglin, A. J. Kellock, H. Rothuizen, and P. Vettiger, Appl. Phys. Lett. 75, 403 (1999). 